\title{
A Systematic Access Through Machine Learning Methods For Expectation In Malady Related Qualities
}

\author{
K.S.S. Joseph Sastry, T. Gunashekar
}

\begin{abstract}
There are Many learning strategies that are been identified with distinguish infection based related qualities. At the early, they as a rule moved toward this issue as a parallel arrangement, where preparing set is involved examples. Examples developed sickness qualities, whereas negative examples are there mining which are not known to be connected with contaminations. This is the essential of the twofold deals based diagrams, since the negative arranging set ought to be true non-infection qualities; regardless, advancement of this set is on a very basic level unfeasible in biomedical inspects. Therefore, to reduce this delicacy, insightfully sensible social gathering based techniques have been proposed. For example, unary outline strategy subject to one-class SVM framework was proposed by grabbing from fundamentally positive models. Also, there mining set may contain cloud torment qualities; as such, semi-formed methodologies, for example, twofold semi-controlled \& positive \& unlabeled (PU) learning blueprints have been proposed. Specifically, PU learning frameworks, which increase from both known suffering qualities \& there mining attributes, were appeared to beat others. In these examinations, information sources are commonly tended to by vectorial plan for cemented classifiers, while they are in bit frameworks for unary \& PU learning ones. The bit based information blend is reasonable for information with various sorts \& it has the majority of the stores of being uncalled for or the relationship subject to various information diagrams. In like manner, in this examination, we looked accumulating structures for the ailment quality figure dependent on vectorial delineation of tests. The spread outcome demonstrated that the unary strategy structure, which joins both thickness \& class likelihood estimation approachs, accomplished the best execution, where as it is most recognizably stunning for the one-class SVM-based technique. fascinatingly, execution of a best twofold outline framework is in each rational sense misty with that of uneven SVM-based PU learning \& twofold semidirected hoarding strategy they are altogether improved.
\end{abstract} characterization; semi-regulated learning; SVM; PU learning strategies; Machine learning

\section{INTRODUCTION}

Ailment quality expectation, a assignment of recognizing a most conceivable competitor illness qualities, is a basic issue in biomedical research. Many learning strategies have been connected to distinguish malady related qualities. In which, a issue is considered as an order issue, where a classifier is found out from preparing information, at that point a educated classifier is

Revised Manuscript Received on August 14, 2019.

K.S.S. Joseph Sastry, Research Scholar, Department of Computer Science and Engineering, (Deemed to be University), Koneru Lakshmaiah Education Foundation, Guntur, A.P, India.

Dr. T. GunaShekar, Associate Professor Department of Computer Science and Engineering, (Deemed to be University), Koneru Lakshmaiah Education Foundation, Guntur, A.P, India.
Keywords :Ailment quality expectation; double order; unary

utilized to anticipate regardless of whether a test/competitor quality is a malady quality. Quickly, at a early, AI based assessments if all else fails progressed toward malady quality need as a twofold mentioning issue, where a learning tests are joined positive preparing tests $\&$ negative sorting out samples[1], for example, Decision Trees (DT)[2,3]k-closest neighbor $(\mathrm{kNN})[4]$, Naive Bayesian classifier[5,6], twofold help vector machine classifier counterfeit neural network(ANN) techniques[10] \& Random Forest(RF)[1]. In ase twofold classifier-based techniques, positive preparing tests are created utilizing known enduring qualities, where as negative engineering tests are there mining which are not known to be related to maladies (thusly, in a succinct minute called cloud properties/set). This is a confinement of parallel classifierbased reactions for tribulation quality check issue, since a negative planning set ought to be veritable non-affliction attributes. Notwithstanding, advancement of this set is about mind blowing in biomedical looks. Along these lines, continually reasonable ways to deal with oversee manage tribulation quality measure have been proposed For example, unary/one class demand, which is found from basically positive models (i.e., acknowledged sickness qualities), has been shown [11-13]. These appraisals utilize done-class SVM proposed by [14] \& piece based information blend structure [15], which is otherwise called a numerous portion adapting (in no time called MKL), to coordinate information from various assets. These unary order based techniques appears the best-fit path to a sickness quality expectation issue, since just a positive preparing set made out of known infection qualities is required for a preparation errand. Be that as it may, truth be told, a obscure set may contain obscure infection qualities; hence, semi-directed learning (SSL) strategies, for example, a twofold semi-administered in like way, PU (i.e., positive \& unlabeled) learning method were proposed to a issue, where a classifiers are found from both labelled(i.e., known affliction qualities) \& unlabeled (i.e., the decrease properties) set. Basic results in these assessments showed that these SSL-based Methods out play out a twofold SVM [7] \& the kNN [4] in like way as a one-class SVM-based framework displayed in [11] \& [13]. As a fore referenced, showing up distinctively in relationship with encouraged assembling based strategies, unary procedure \& SSL-based ones, particularly PU learning, appears to be progressively sensible for a illness 


\section{A SYSTEMATIC ACCESS THROUGH MACHINE LEARNING METHODS FOR EXPECTATION IN MALADY RELATED QUALITIES}

quality forecast. Be that as it may, the execution examination of them done by past investigations depended on various information portrayal of qualities. For example, the correlation between PU learning \& unary portrayal strategy was done on area based outline of data sources where as vector based system was used for a assessment between facilitated approach based \& PU learning methods. Additionally, a bit based data mix is sensible for a data of different sorts since it facilitates a best resemblance figuring methodology for every sort of data by changing evident data structures (i.e., vectors, strings, trees, traces, etc.) into part systems. In like manner, when various features (i.e., high-dimensional data)are used to delineate properties, a bit based data mix can be profitable since deficient mode can be academic with L1-standard MKL advance strategies[15]. A despairing condition is colossal to see appropriate sources from countless information sources. In any case, in biomedical applications, there are typically few sources \& a large portion of these information sources are cautiously chosen \& pre-handled. Conversely, vector-based information combination technique is less complex, since every datum source is spoken to by an element of a component vector. Also, a significance of every datum source is normally indicated by a learning calculation, however not reliant of a improvement systems utilized in a part based information combination strategies. In addition, a vectorbased information combination procedure can be utilized in any learning procedures (i.e., bit based, for instance, SVM \& non-bundle based, for instance, Naïve Bayesian \& $\mathrm{RF})$.

In this evaluation, we consider a speculation execution of facilitated amassing based, unary system based \& SSLbased procedures for a sickness quality need issue subject to vectorial depiction of data. In particular, we picked RF, which is a best united procedure as showed up in our past assessment [1] for a misery quality check. For unary portrayal based structure, we in like way used one-class SVM technique as past appraisals. Also, we tried an ovel unary technique one proposed by which joins both thickness/stream \& class probability estimation techniques. For SSL-based systems, we rehashed indistinguishable technique to develop preparing set from in however dependent on an alternate parallel semi managed learning strategy. Moreover, two PU learning strategies, which dependent on one-sided \& staggered weighted SVMasin were utilized. A reproduction results demonstrated that a unary solicitation proposed by accomplished a best execution, where as it was most discernibly horrible for a one-class SVM-based strategy. Strikingly, execution of RF is in every way that really matters unclear with that of a uneven SVM-based PU learning \& a parallel semi coordinated solicitation strategies. In like manner, they are unmitigated improved than a staggered SVM-based one.

\section{DATA PREPARATION \& LEARNING METHODS}

A. Classification frameworks, parameter settings \& execution

\section{a. Binary portrayal approach}

In this assessment, we picked a best parallel methodology framework for tribulation quality need as we showed up in our past appraisal [1] that is Random Forest. This is a social affair winning strategy for sales that works by structure an oar decision trees achieving time. For parameter settings \& use, we used default settings for RF in WEKA.

\section{b. Unary arrangement strategies}

For arrangement strategies, we utilized one-class SVM [14] \& another class methodology showed in. Specifically, one-class SVM proposed by [14] was completely utilized in illness quality need. A principal system is to discover a hyper-plane that isolates the vector delineations of the weight attributes from a most dependable starting stage create with a most ideal edge, $\&$ recognize a quality bound to be a sullying quality on a off chance that it lies continually remote toward this hyper-plane. an, a strategy proposed by sets both thickness/task \& class likelihood estimation structures. To a degree anyone is concerned, this methodology has not yet been utilized for a torment quality figure issue. For parameter settings \& execution, we utilized default settings for one-class SVM in Lib SVM \& WEKA pack for a technique proposed by.

\section{c. Binary_semi-supported gathering system}

In _this evaluation, _we utilized a direct _semi controlled _SVM system (ShortlycalledLS3VM). For parameter settings \& execution, we utilized default settings for this strategy in SVM lin pack (http://vikas.sindhwani.org/svmlin.html).

\section{d. PU learning frameworks}

For PU learning frameworks, we picked an uneven SVM as in ProDiGe \& a dazed weighted SVM as in PUDI More explicitly, we utilized a uneven SVM classifier to over-loads positive perspectives amidst proposing to address a way wherein that they address high-sureness models where as a "negative" models are known to contain false negatives, which they ought to find. Meanwhile, for a stupified weighted SVM-based technique, the unlabeled set is isolated into a couple of named ones subject to how much a decrease model is basic to positive ones, by then a puzzled weighted SVM classifier is attempted to see novel positive models from unlabeled ones. For parameter settings \& execution of a set SVM-based techniques, we utilized LibSVM with default settings \& picked a best mix of class loads for each structure.

\section{B. Building a list of capabilities}

To construct a list of capabilities, we gathered entomb nuclear, genomic \& proteomic information for 
qualities/proteins. Specifically, a protein cooperation arrange comprising of 13,488 proteins \& 217,893 communications was gathered from Interolog collaboration database (I2D) \& used to figure topological highlights of proteins, for example, degree, 1-Nindex, 2Nindex, separation to ailment qualities \& positive topology coefficient. These topological properties were seen about their factually noteworthy contrast between two gatherings of ailment \& non-infection qualities [4]. Furthermore, it was accounted for that proteins with longer arrangement may give greater chance to transformation $[2,3]$ \& explicit quality cosmology terms may a not at e ailment data of proteins ; along these lines, we gathered succession information \& quality ontology(GO) terms of proteins from UniProtKB to ascertain protein length \& number of straightforwardly a recorded GO terms, separately. Besides, contemplate in demonstrated that more spaces \& restricting locales may enable transformation to all a more effectively degenerate protein capacities. In view of this perception, we utilized an information base of protein, InterPro by means of BioMarttool to ascertain number of spaces $\&$ number of restricting destinations for every protein. Last, in view of a perception that advancement rate of quality may add to a probability of here ditary infection [3], we utilized an information base of homolog qualities, NCBI's Homologene to pick a advancement pace of every protein. In structure, a total of ten highlights including topological, advancement, urgent, explanation, \& developmental properties of every protein we renamed (seeTable1). They are everything seen as numerical information \& coordinated in a degree of $[0,1]$ for all assessments in this paper.

\section{Design of a course of action set}

A positive preparing tests relied on known torment attributes assembled from OMIM. In a wake of gettogether basically relating proteins open in a protein made effort plan, we acquired 2,365 known defilement proteins. This set was played as positive arranging set (P). Remaining proteins $(\mathrm{R})$ in a protein association sort out are proteins which are not known to be connected with an ailment. This set wires cloud ruining proteins, non-tainting proteins \& 2,056 noteworthy proteins. These central proteins were assembled from three information sources DEG, BioMart \& DAVID . As indicated by consider , a essential proteins have commonly striking properties stood out from unsettling influence \& non-contamination proteins. In this way, were move basic proteins from $\mathrm{R}$ to plot a unlabeled set (U) of 11,123 proteins

\section{a. Binary course of action methodology}

For this framework, a course of action set wires positive $\&$ negative preparing tests with shady size from managed in past joined outline based methods. Consequently, a positive preparing set joins most of a 2,365 known illness proteins $(\mathrm{P})$, where as a sporadic set (RS) of identical sizes investigated a unlabeled set (U) was played as a negative arranging samples(N).

\section{b. Unary strategy systems}

For unary strategy system, fundamentally set $\mathrm{P}$ was utilized to structure classifiers.

\section{c. Binary semi-oversaw depiction methodology}

We scanned for after a similar approach proposed by to accumulate a status set. Specifically, it joins positive $(\mathrm{P}) \&$ negative $(\mathrm{N})$ engineering sets as in a joined storing up structure. Moreover, a sum of 7,374 neighbors of distress proteins in a human protein joint effort orchestrate from $\mathrm{U}$ were picked as unlabeled models (UP) for arranging.

\section{d. $\quad$ PU learning frameworks}

For PU learning systems, both P \& U were utilized for arranging. Specifically, we utilized a two PU learning methods as above-showed up. For the uneven SVM-based method, a self-decided set (RS) of negative organizing tests were inquired about $U$ with not very much described size from a positive preparing set $(\mathrm{P})$. This framework is equivalent to that improved a situation a parallel procedure approach. Regardless, an uneven SVM was utilized to over-loads positive perspectives amidst a strategy thinking. For a stupified weighted SVM-based philosophy, a set $\mathrm{RS}$ was appropriated to four checked sets (i.e., dependable negative set (RN), likely positive set (LP), likely negative set $(\mathrm{LN})$, \& frail negative set $(\mathrm{WN})$ ) in light of how much a reduce quality is crucial to torments of intrigue an offense PUDI .

\section{Execution Assessment}

So as to assess $\&$ consider these depiction systems for a malady quality guess, we utilized 10-overlay crossunderwriting on a preparation set \& evaluated a execution by exactness. For a systems, which use tests arbitrarily perused obscure quality set, to additionally abstain from examining predisposition \& to lessen the execution change we rehashed this inspecting technique 100times. At that point, a classifier was prepared \& assessed for each time, lastly a general execution of every technique was found a middle value of over correctness's.

\section{RESULTS}

\section{Perfect burdens for SVM-based PU learning systems}

For the PU learning system which depends on an uneven SVM classifier, we locate a best classifier by moving a degree of weight between a positive set $(\mathrm{P}) \&$ a optional set $(\mathrm{RS})$ in a range $[1,2]$. Figure 1 exhibits that the structure accomplished best execution for turmoil quality check with a degree is 1.1.For a stifled SVM-based methodology, we utilized a best setting of burdens (i.e. the stores for P, LP, WN, LN, RN are 1.5, 1, 1, 1.1, 1.2, freely) 


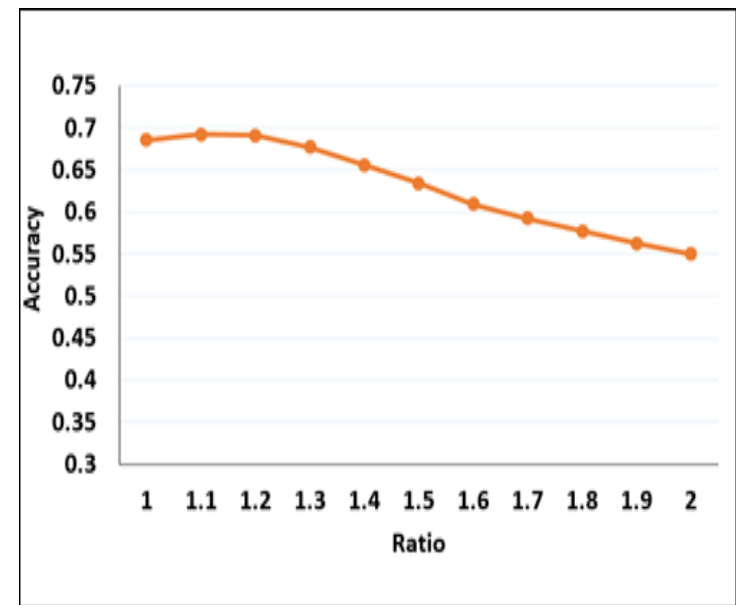

Figure1._Performance of_the one-sided_SVM-PU learning strategy.

\section{Performance correlation}

As previously mentioned, the execution of every strategy depends on precision. This was determined utilizing 10-overlap cross-approval on a preparation set. Likewise, it is found a middle value of significant worth more than 100 preliminary for strategies which require irregular arrangement of tests from a unlabeled set. Figure 2 demonstrates a execution of a including

I) a parallel depiction method (i.e.,RF)

ii) two unary social occasion strategies (i.e.,one-class SVM \& one class Hempstalk which was proposed in);

iii) a coordinated semi-composed solicitation methodology

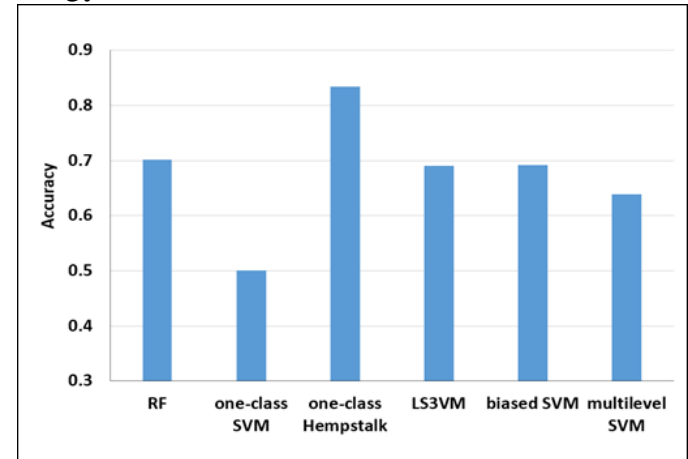

Figure 2.Performance correlation of a techniques for illness quality expectation

(i.e.,LS3VM) \& iv) two PU learning techniques (i.e., uneven SVM-based \& flabbergasted SVM-based ones). Unmistakably a one-class strategy proposed by achieved best execution (i.e., accuracy $=0.83$ ), while it is a most exceedingly repulsive for a one-class SVM (i.e.,accuracy $=0.5$ ). This result is facilitated with results showed up on UCI educational conglomerations. There a tyke forward is unavoidable execution is that a one-class SVM proposed by [14] is fundamentally chosen class probability estimation procedure, where for what it's value additionally chose thickness/transport frameworks in. These two frameworks are principle answers for one-class learning figurings. Moreover, it was showed up in that a stunned SVM-based PU learning system crushed a procedure which relies on uneven SVM. Notwithstanding, it is exchange in our assessment results (i.e., accuracy $=0.69 \& 0.64$ for uneven \& stunned SVM,
respectively).This partition may be acknowledged by different in the used dataset. The execution of LS3VM (for instance accuracy $=0.69$ ) was equivalently better than that of the stunned SVM-based structure. This is in light of the way that a certification of unlabeled models for getting ready in LS3VM is unendingly reasonable. In all actuality, these unlabeled models are proteins which are quick interface ants of known disease proteins. This choice depended on an "infection Module" standard, which have been broadly utilized in system based strategies for sickness quality forecast (i.e., qualities/protein related with the equivalent/comparative illness will in general find nearly in quality/protein communication organize). Mean while, parcel of unlabeled models in the emotional set (RS) in a astonished SVM system is to change a ailment quality figure to multi-class game-plan with weights doled out to every individual class. Much more strikingly, RF accomplished second best execution. It was the best one showed up contrastingly in connection to other coordinated assembling systems for pollution quality want [1]. This outcome also infers the power of get-together framework for course of action issues. unequivocally, it has beginning late proposed a troupe technique for PU learning \& appeared to crush all other game-plan based strategies for the ailment quality want issue.

\section{CONCLUSION}

Machine learning-based examinations to take care of a issue of distinguishing malady related qualities have considered this issue as a illness quality forecast, in which an arrangement show is first consequently gained from a preparation information \& afterward used to dole out obscure qualities to the infection or non-ailment quality class. A well known figuring out how to-arrange approach is double order, which requires both positive (sickness qualities) \& negative (non-illness qualities) preparing occurrences. In any case, this necessity is unfeasible, taking into account that in biomedical research we can't ensure that some quality isn't identified with a given infection; as such, non on-sickness qualities are accessible so we can utilize a mass negative preparing occasions. Other figuring out how to-order strategies that are progressively reasonable (when not basically requiring negative preparing occurrences) incorporate one-class \& PU learning. Where as one-class learning utilizes basically positive preparing occasions, PU learning utilizes both positive sorting out occasions \& unlabeled information (i.e., attributes not known to be connected with a given affliction). Past assessments have demonstrated that semimade strategies (checking PU learning) are superior to oneclass \& parallel deals frameworks. In this assessment, in setting on vectorial portrayal of test information, we showed that a unary approach structure, which affiliation both thickness \& class likelihood estimation system, accomplished a best execution, where as it is most exceedingly nefarious for a one-class SVM-based structure. Particularly, execution of a best twofold solicitation 
structure is proportionate to that of uneven SVM-based PU learning \& twofold semi-controlled systems. Besides, they are everything seen as improved than a perplexed SVM-base done.

\section{REFERENCES}

1. D. H.Le,N. Xuan Hoai, and Y.-K. K won," A Comparative Study of Classification Based Machine Learning Methods for Novel Disease Gene Prediction," Knowledge and Systems Engineering, Advances in Intelligent Systems and Computing V.-H.Nguyen, A.C.LeandV. -N.Huynh, eds., pp. 577-588: Springer International Publishing, 2015.

2. N.Lospez-Bigas, and C.A.Ouzounis, "Genome-wide identification of genes likely to be involved in human genetic disease," Nucleic acids research, vol.32, no.10, pp.3108-3114, 2004.

3. E.Adie, R.Adams, K.Evans, D.Porteous, and B.Pickard, "Speeding disease gene discovery by sequence based candidate prioritization," BMC Bioinformatics, vol.6, no.1, pp.55, 2005.

4. J.Xu, and Y.Li, "Discovering disease-genes by topological features in human protein-protein interaction network," Bio informatics, vol.22, no.22, pp. 2800-2805, November15, 2006, 2006.

5. S.Calvo, M.Jain, X.Xie, S.A.Sheth, B.Chang, O.A.Goldberger, A.Spinazzola, M.Zeviani, S.A.Carr, and V.K.Mootha, "Systematic identification of human mitochondrial disease genes through integrative genomics," NatGenet, vol.38, no.5, pp.576-582, 2006.

6. K.Lage, E.O.Karlberg, Z.M.Storling, P.I.Olason, A.G.Pedersen, O.Rigina, A.M.Hinsby, Z.Tumer, F.Pociot, N.Tommerup, Y.Moreau, and S.Brunak, "A human phenome-interactome network of protein complexes implicating genetic disorders," NatBiotech, vol.25, no.3, pp.309-316, 2007.

7. A.Smalter, S.F.Lei, and X.-w.Chen," Human diseasegene classification with integrative sequence-based and topological features of protein-protein interaction networks." pp.209-216.

8. P.Radivojac, K.Peng, W.T.Clark, B.J.Peters, A.Mohan, S.M.Boyle, and S.D.Mooney, "An integrated approach to inferring gene-disease associations in humans," Proteins: Structure, Function, and Bioinformatics, vol.72, no.3, pp.1030-1037, 2008.

9. S.Keerthikumar, S.Bhadra, K.Kandasamy, R.Raju, Y.L.Ramachandra, C.Bhattacharyya, K.Imai, O.Ohara, S.Mohan, and A.Pandey, "Prediction of candidate primary immuno deficiency disease genes using asupport vector machine learning approach," DNA Research, vol.16, no.6, pp. 345-351,2009.

10. S.Jiabao, J.C.Patra, and L.Yongjin, "Functional Link Artificial Neural Network-based disease gene prediction."pp. 3003-3010.

11. T.DeBie, L.-C.Tranchevent, L.M.M.VanOeffelen, and Y.Moreau, "Kernel-based data fusion for gene prioritization," Bio informatics, vol.23, no.13, 2007.

12. S.Yu, S.VanVooren, L.-C.Tranchevent, B.DeMoor, and Y.Moreau, "Comparison of vocabularies, representations and ranking algorithms for gene prioritization by text mining," Bio informatics, vol.24, no.16, pp.i119i125,2008.

13. S.Yu,L.-C.Tranchevent, B.DeMoor, and Y.Moreau, "Gene prioritization and clustering by multi-view text mining," BMC Bio informatics, vol.11, no.1, pp.28,2010.

14. B.Schölkopf, J.C.Platt, J.Shawe-Taylor, A.J.Smola, and R.C.Williamson, "Estimating the support of a highdimensional distribution," Neural computation, vol.13, no.7, pp.1443-1471, 2001.
15. G.R.G.Lanckriet, N.Cristianini, P.Bartlett, L.E.Ghaoui, and M.I.Jordan, "Learning the kernel matrix with semi definite programming," The Journal of Machine Learning Research, vol.5, pp.27-72, 2004.

\section{AUTHORS PROFILE}

K.S.S. Joseph Sastry Research Scholar, Department of Computer Science and Engineering, (Deemed to be University), Koneru Lakshmaiah Education Foundation, Guntur, A.P, India.

Dr. T. GunaShekar Associate Professor Department of Computer Science and Engineering, (Deemed to be University), Koneru Lakshmaiah Education Foundation, Guntur, A.P, India. 